to England reduction was again attempted under an anæsthetic with block and tackle. He was admitted into the Seamen's Hospital, Greenwich, more than three months after the accident, with the left lower limb considerably flexed and everted, the pelvis tilted and buttock flattened. The thigh could be flexed on the abdomen but could not be extended. Both internal and external rotation were limited to less than half their extent. The head of the femur could be felt almost in the perineum and the great trochanter about the centre of the buttock. There was shortening of one and a half inches, and when standing upright the great toe of the left foot did not quite touch the ground, the heel being drawn up and the foot everted (Fig. 1). The patient could only limp and hobble about with a stick. The head of the bone, which was found lying to the inner side of the obturator foramen, was excised on Feb. 18th, 1896. Several contracted bands of fascia and muscle in front of the thigh required division before the limb could be straightened. The head of the femur was found to be denuded of cartilage for about half its extent, and there had obviously been considerable damage done to the parts round the joint by the accident and the previous attempts at reduction. The present condition of the patient is satisfactory. He is able to walk about all day without pain or tiring and his leg is straight with his body. There is a little over two inches shortening, the limbs measuring thirty-six and a half inches and thirty-four and a half inches respectively (Fig. 2).

FIG. 2.

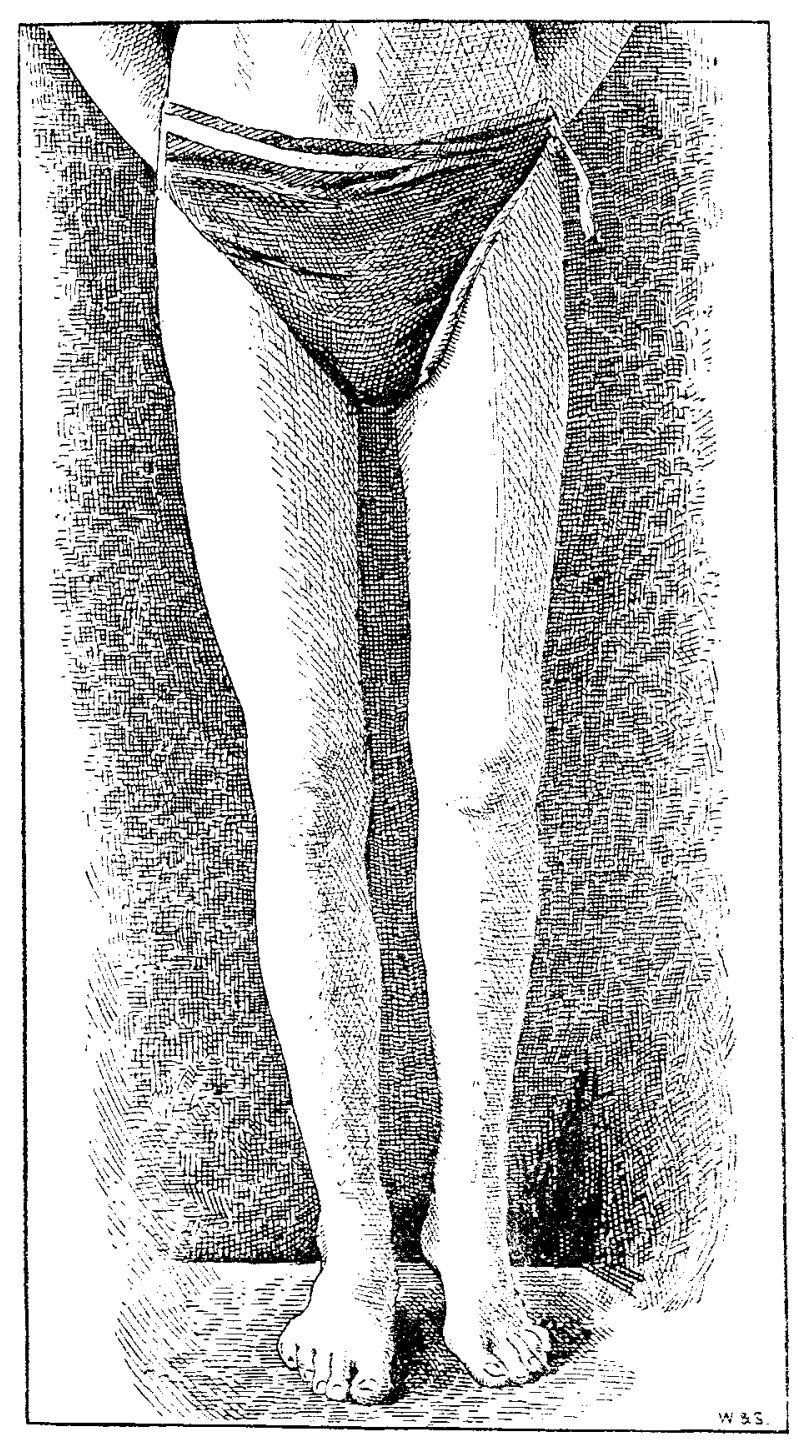

The position after operation. The patient stands alone and is very little inconrenienced by the two inches of shortening.

The scars in the perineum and groin are quite healthy. There is no movement at the hip and practically no eversion when the patient is lying down, though he throws his foot outwards a little when walking.

Green-street, Park-lane, W.

Medical Magistrate.-Mr. John R. Fox, M.B. Melb., has been appointed a Justice of the Peace for the Southern Bailiwick, Victoria, Australia.

\section{A CASE OF SCARLET FEVER TREATED BY ANTI-STREPTOCOCCIC SERUM.}

BY A. KNYVETT GORDON, M.B., B.C., B.A. CANTAB., ASSISTANT MEDICAL OFFICER, SOUTH-EASTERN FEVER HOSPITAT (METROPOLITAN ASYLUMS BOARD).

A VIGoRous boy aged six years was admitted to the South-Eastern Fever Hospital on Nov. 17th, 1896, certified as suffering from scarlet fever. There was a history of vomiting and sqpe-throat on Nov. 14th, "rash all over" on the 15th, and nasal discharge on the 16th. On admission the boy was semi-comatose, the face was pale, and the respiration stertorous; the trunk and limbs were covered with a characteristic punctiform eruption, which was crimson in the groins but elsewhere dusky-almost purple, in fact; there was commencing desquamation on the face ; the pulse was only just perceptible at the wrist and could not be counted. The tonsils and palate were much swollen and brightly injected; there was some viscid bloodstained mucus covering the mouth and fauces, but when this was cleared away the tonsils were seen to be free from any deposit. The temperature was $104^{\circ} \mathrm{F}$. He was ordered milk and eggs only, with two ounces of brandy in the twenty-four hours. On the next day (Nov. 18th) the temperature varied from $103^{\circ}$ to $101 \cdot 5^{\circ}$, the pulse was very soft (140), and there was superficial ulceration of both tonsils, but less swelling and no deposit or sloughing. The heart and lungs were normal. On the 19th the temperature was $103^{\circ}$; the rash was not so dusky, and there was slight general improvement, the patient taking milk more willingly. There were well-marked urticarial wheals on the chest. On the 20 th the child was much worse; the rash was very dusky, the fauces were dry and offensive, the ulceration was very slight, there was less. swelling, and a little soft deposit on the ulcerated surfaces. The temperature was $103^{\circ}$ and the pulse was 180 . The heart was dilated to two fingers' breadth outside the nipple line and to mid-sternum; the first sound was very faint and there: was no murmur. A fresh specimen from the fauces showed streptococci and staphylococci, no bacilli at all, and none on cultivation. There was diarrhoea. Ten c.c. of the sérum antistreptococcique of the Institut Pasteur were injected under the skin of the abdomen at 5 P.M. The brandy was increased to three ounces. On the 21 st the temperature was $103^{\circ}$ and the pulse 106 and much stronger; the fauces were less offensive, and there was less swelling. The heart extended from mid-sternum to just outside the nipple line; there was a better first sound. Another 10 c.c. of the serum were given at 12 noon. On the 22 nd the temperature was $99.5^{\circ}$; the fauces were clean and not offensive; there was very slight swelling; the pulse was 96 and of much better tension. There was no urticaria, the scarlet rash was fading, and there was free desquamation. On the 23rd the temperature was $98^{\circ}$ and the child was intelligent, answering questions, and hungry. He was ordered bread-and-milk and the brandy was omitted. On the 24th the left membranum tympani was red; there was a small perforation in the anterior inferior segment discharging odourless pus. The temperature was normal. $\mathrm{He}$ was ordered bread-and-butter and pudding. On the 28th he was sitting up in bed and had fish diet. On Dec. 2nd he was given mince diet. On the 3 rd he was up in blankets and on the 12th he was up in clothes.

This case has seemed to me to be worth publishing on account of the rapidity with which the improvement followed the administration of the serum. On admission the child seemed moribund, but he rallied from the effects of the journey in the ambulance and commenced to improve a little till the serenth day of the disease; then he grew rapidly worse and was to all appearances dying from septicæmia. He was semi-comatose, with a failing heart, dusky rash, and diarrhœa. After one dose he rallied, and after the second a rapid improvement took place and was maintained, his convalescence occurring very much sooner than is usually the case with patients who have had any septic symptoms.

With regard to the indications for the use of the serum in scarlet fever, I would suggest that it would be valuable in any case that becomes septic, whether the signs exist from the first and are not in proportion to the severity of the throat symptoms-as in this case, where the throat was not 
severely affected - or whether the condition is due to absorption from a sloughing throat or nose-the more common occurrence. In the latter case improvement often begins when the throat is vigorously attacked by constant swabbing with chlorinated soda or some other germicide, but I do not know of anything that has much effect when there is septicæmia with a moderately severe or even a mild throat. In this case the only drug given was brandy, and I do not think the remarkable improvement could be attributed to the stimulant only.

I am indebted to Dr. F. M. Turner, medical superintendent of the hospital, for permission to publish the case.

\section{A NEIV METHOD FOR LOCALISING BRAIN LESIONS.}

BY ROBERT H. COX, F.R.C.S. IREL., SURGEON TO THE IMPERIAL MARITIME CCSTOMIS, CHINA.

THAT there is need for a more perfect method of locating the important areas of the cerebral cortex on the overlying surface of the scalp will, I think, be admitted by all, seeing that most of, if not all, the methods generally employed are either limited in their scope or complicated in their application, and many give rise to error by the use of a standard measure-the inch or the centimetre-for varying surfaces. The method which I am about to describe will, I think, be found devoid of these objections. For its use two things are necessary-viz., (a) an instrument which I have invented and called (for want of a better name) a cerebro-graphometer; and (b) a diagrammatic map of a hemisphere of the brain, prepared from readings made by the use of the same instrument on the cadaver and on casts of the brain in situ. This latter may, however, be replaced or augmented by a list of indices made in the same way.

[The author has prepared such a "list of indices," showing in much detail the correspondence between the letters and figures marked on the instrument and various regions of the brain-namely, motor centres (nine in number), sensory centres (hearing and sight), special convolutions (fourteen in number), special fissures (those of Rolando and Sylvius), the middle meningeal artery, and the cerebellum.]

The instrument itself consists entirely of the mechanical device known technically as "lazy tongs" formed into a circle with two accessory loops. The circle or base contains twenty-eight fenestræ and has attached to it

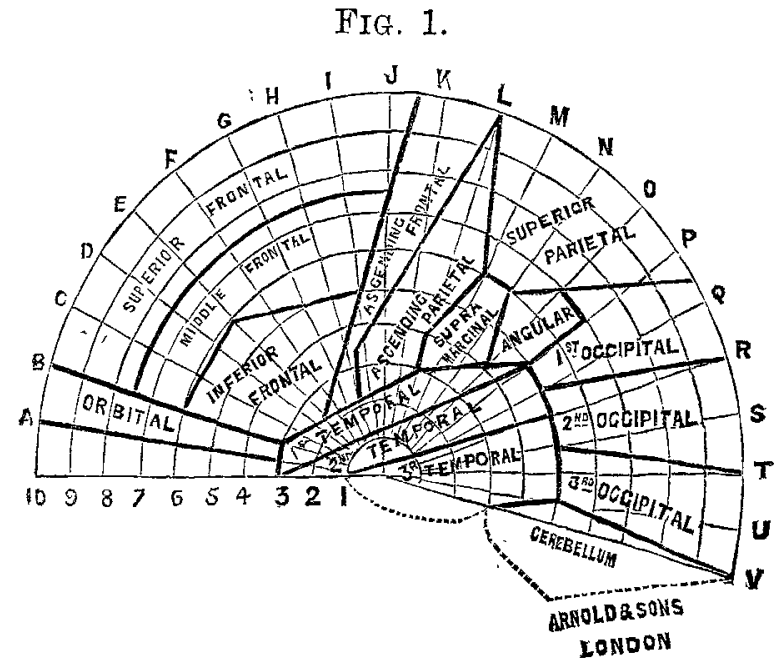

A diagrammatic map (on a gnomonic projection) of the external surface of a hemisphere of the brain, showing the external surface of a hemisphere of the brain, showing the boundaries of the lobes and convolutions and the lines of of the instrument.

externally at four equi-distant parts the two accessory loops by their end rivets (excepting at one place, where the attachment is at the rivet third from the end) in such a way that the loops arch over the circle at right angles to each other, while at the same time they are capable of a hinge-like movement. One loop bears on its central rivets the numerals, beginning at each end with 1 and ending in the middle with 10 ; while the other loop bas the letters $A$ and $V$ in consecutive order from before backwards, the rivet marked $\mathbf{T}$ being that connecting the loop with the circle behind. The map (Fig. 1) is made on a gnomonic projection with the radii, or longitudinal lines, marked by letters, and the semicircles, or lines of latitude, by numerals, both of which correspond with the engraved

FIG. 2.

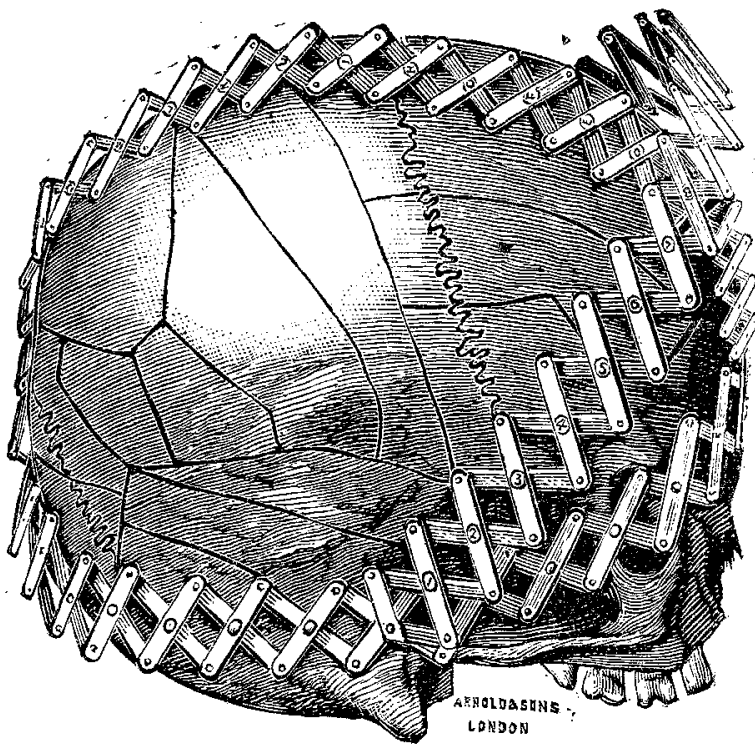

The regions enumerated in Fig. 1 are here projected on the right side of the skull. The illustration also shows the instrument in position for localising.

loops of the instrument. Fig. 2 represents the instrument in position for localising.

To use the instrument it is necessary to extend it in all parts and apply it to the human head, with the rivet forming the junction of the lettered loop with the circle (below that marked " A") on the glabella and the rivet marked " $V$ " (at the extreme end of the same band) to the occipital protuberance, when the loop between these two points should be pressed down to the skull in the middle line; while the circle is also closed on the skull on such a plane that the central rivet, marked 10 , of the numbered loop shall lie on the middle line, as shown by the lettered loop. The instrument is thus fixed to the head by the circular band and the lettered loop, while the numbered loop is capable of moving backwards and forwards with a hingelike motion, during which motion each numbered rivet describes a semicircle of latitude, and the portion of the same band from one of the fixed rivets to the numeral 10, as it extends to each letter, will gire the radii of longitude. With the instrument thus in position, to finc any given region-say, Broca's lobe-it is only necessary to consult either the map (Fig. 1) or the list of indices for the bearings there given, as $\frac{\mathrm{C}}{4}$ (left side), when by moving the 10 of the numbered loop forward to " $\mathrm{C}$ " and pressing it down on the scalp the numeral " 4 " will lie over the part. Rolando"s fissure may be found in a similar way by locating each end and joining the two points by a straight line. Localising by this method may be thus summed up: 1. Application of the apparatus. Extend the instrument and apply the end of the lettered loop marked " $\mathrm{V}$ " to the occipital protuberance and the other end to the glabella, then press down the loop to the scalp in the middle line. Close the circle round the head so that the 10 on the numbered loop will lie on the lettered loop. 2. Location of part sought for. Consult either the map or the list of indices for the bearings. Place the number 10 on the letter of longitude and press the numbered loop down, when the number of latitucle will rest over the part sought for.

Having thus shown the simplicity of the method, for the carrying out of which no recourse need be had to the science of mathematics in any of its branches, and the almast automatic action of the cerebro-graphometer, I would now ask attention while I try to explain its accuracy. As may be seen at a glance, the instrument is equally applicable to all sized heads and forms its own unit of measurement for each, thus eliminating the errors due to the use of standard measure for a varying surface. The two fixed points taken are easily found and are those which have proved most reliable-the glabella and the occipital protuberance. The circular band is placed on that plane of 